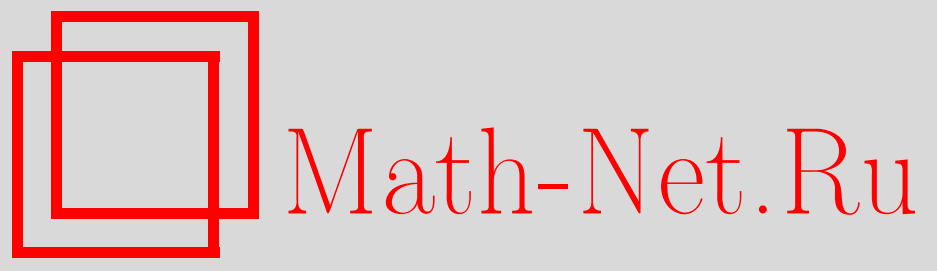

А. С. Рыбаков, Спектральные свойства линейного конгруэнтного генератора в специальных случаях, Дискрет. матем., 2004, том 16, выпуск 2, 54-78

DOI: https://doi.org/10.4213/dm152

Использование Общероссийского математического портала Math-Net.Ru подразумевает, что вы прочитали и согласны с пользовательским соглашением http://www . mathnet.ru/rus/agreement

Параметры загрузки:

IP : 3.80 .253 .173

26 апреля 2023 г., $07: 23: 00$

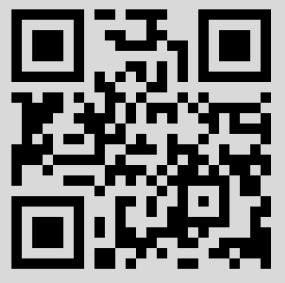


УдК 519.7

\title{
Спектральные свойства линейного конгруэнтного генератора в специальных случаях
}

\author{
() 2004 г. А. С. Рыбаков
}

В данной работе для линейного конгруэнтного генератора

$$
z_{N+1}=G\left(z_{N}\right), \quad N=1,2, \ldots,
$$

где $G(x)=\lambda x+c(\bmod W), W=p^{F}, p-$ простое число, имеющего полный период и множитель $\lambda$ специального вида, устанавливается нетривиальная нижняя оценка наименьшего ненулевого волнового числа $e_{L}(\lambda)-$ базовой характеристики, вводимой в спектральном тесте для проверки случайности на основе изучения частоты встречаемости $L$-наборов $\left(t_{1}, \ldots, t_{L}\right)$ в последовательности $\left(z_{N}\right)$.

Указываемая нижняя граница имеет вид $W^{1 / L-\delta}$, где $\delta$ - некоторая величина, явно зависящая от параметров, определяющих множитель $\lambda$. При специальном выборе параметров $\delta$ можно сделать сколь угодно малым. Показатель $1 / L$ в этой оценке нельзя заменить большим. Такого рода оценки необходимы для изучения классов множителей, проходящих спектральный тест.

\section{1. Введение}

Для построения последовательности $\left(\zeta_{N}\right)$ псевдослучайных чисел (см. гл. 3, п. 3.1 в [3]), отвечающих равномерному распределению на промежутке $[0,1)$, на практике часто прибегают к следующей простой конструкции.

Пусть $W$ - фиксированное большое натуральное число. (Его величина может достигать нескольких сотен или тысяч бит). С точки зрения практики наибольший интерес представляет случай, когда $W$ равно степени 2 . В данной работе мы будем предполагать, что $W=p^{F}$, где $p-$ простое число, $F \in \mathbf{Z}_{\geqslant 2}-$ растущий параметр. Здесь и далее $\mathbf{Z}_{\geqslant m}=\{m, m+1, \ldots\}$.

Пусть $\left\{G_{\lambda, c}\right\}$ - семейство линейных конгруэнтных генераторов по модулю $W$, то есть отображений множества $I_{W}=\{0,1, \ldots, W-1\}$ в себя таких, что

$$
G_{\lambda, c}(x) \equiv \lambda x+c \quad(\bmod W)
$$

где $\lambda$ и $c$ - произвольные натуральные числа. Сравнимые между собой пары индексов $(\lambda, c)$ определяют один и тот же линейный конгруэнтный генератор, поэтому, не ограничивая общности, можно считать, что $\lambda$ и $c$ - также элементы множества $I_{W}$. 
Фиксируя какие-либо числа $z_{0} \in I_{W}, \lambda \in I_{W}$ и $c \in I_{W}$, можно построить псевдослучайную последовательность элементов $I_{W}$

$$
z_{0}, z_{1}, z_{2}, \ldots
$$

такую, что $z_{N+1}=G_{\lambda, c}\left(z_{N}\right), N=0,1,2, \ldots$, с помощью которой в итоге определяется последовательность $\left(\zeta_{N}\right)$ :

$$
\zeta_{N}=z_{N} / W, \quad N=0,1,2, \ldots
$$

В данной работе мы будем рассматривать лишь последовательности (3), имеющие период максимальной длины $W$. Необходимым и достаточным условием для этого в силу п. 3.2.1.2 гл. 3 в [3] являются условия $p \nmid c$ и

$$
\lambda=1 \quad(\bmod p) \quad(\text { при } p>2), \quad \lambda=1 \quad(\bmod 4) \quad(\text { при } p=2) \text {. }
$$

Естественно поставить вопрос о проверке последовательности (3) на близость к случайной. Здесь и ниже под случайной последовательностью имеется в виду выборка из последовательности независимых равномерно распределенных на промежутке $[0,1)$ случайных величин. Последовательность считается близкой к случайной, если она успешно проходит определенный набор статистических тестов на случайность, например, тех, что описаны в п. 3.3 главы 3 книги [3].

Наиболее совершенным, по мнению Д. Кнута, из имеющихся тестов на случайность является спектральный тест (см. п. 3.3 .4 гл. 3 в [3]), предложенный в 1965 г. Р. Ковэю и Р. Макферсоном. Вероятно, будет уместным привести соответствующую щитату из [3], показывающую важность этого теста:

“Этот тест замечателен тем, что все известные плохие датчики, основанные на линейном конгруэнтном методе, были им забракованы, в то время как все хорошие датчики прошли его удовлетворительно. Безусловно, это наиболее совершенный из имеющихся тестов, в связи с чем он заслуживает особого внимания."

Для определенности под близостью последовательности к случайной в данной работе имеется в виду успешное прохождение именно спектрального теста.

Пусть $L-$ фиксированное натуральное число. Спектральный тест производит проверку на случайность посредством исследования преобразования Фурье функщии $\left(t_{1}, \ldots, t_{L}\right) \mapsto H\left(t_{1}, \ldots, t_{L}\right)$, где $H\left(t_{1}, \ldots, t_{L}\right)$ - частота появлений набора $\left(t_{1}, \ldots, t_{L}\right)$ в последовательности (2) на отрезке длины, равной длине ее периода. При этом существуют две формы спектрального теста (сильная и слабая), для каждой из них результат проверки определяется по значению наименьшего ненулевого волнового числа

$$
e_{L}(\lambda)=\min \left\{\sqrt{h_{1}^{2}+\ldots+h_{L}^{2}}: \mathbf{0} \neq\left(h_{1}, \ldots, h_{L}\right) \in \mathbf{Z}^{L}, \sum_{i=1}^{L} h_{i} \lambda^{i}=0 \quad(\bmod W)\right\} .
$$

Выписанное только что выражение имеет силу лишь для последовательностей (3) периода максимальной длины.

Для спектрального теста в слабой форме мерой близости к случайности принято считать величину $1 / e_{L}(\lambda)$. Допустим, мы хотим потребовать, чтобы первые $l p$-ичных разрядов чисел (3) были, условно говоря, случайны, где $l$ - фиксированное натуральное число. Спектральный тест в этом случае будет пройден, если выполнено неравенство $1 / e_{L}(\lambda) \leqslant p^{-l}$, то есть $e_{L}(\lambda) \geqslant p^{l}$. 
Спектральный тест в сильной форме требует выполнения неравенства

$$
\gamma_{L}(\lambda)=\frac{\pi^{L / 2}\left(e_{L}(\lambda)\right)^{L}}{\Gamma(L / 2+1) W} \geqslant 0,1,
$$

где $\Gamma(z)$ - гамма-функция. Отметим, что граница 0,1 здесь установлена лишь на основании опытных данных и ее выбор можно считать относительно произвольным.

Неравенство (5) следует интерпретировать геометрически. Смысл его состоит в том, что наименьший ненулевой вектор решетки

$$
\left\{\left(h_{1}, \ldots, h_{L}\right) \in \mathbf{Z}^{L}: \sum_{i=1}^{L} h_{i} \lambda^{i}=0 \quad(\bmod W)\right\}
$$

не может быть слишком мал. Объем $L$-мерного шара радиуса $e_{L}(\lambda)$ (где $e_{L}(\lambda)$ - длина этого вектора) должен составлять не менее 1/10 от объема ее основного параллелепипеда.

Заметим, что это довольно жесткое условие. С ростом $L$ оно влечет неравенство

$$
e_{L}(\lambda) \geqslant W^{1 / L} \sqrt{\frac{L}{2 \pi e}}(1+o(1)),
$$

в то время как в силу теоремы Минковского о выпуклом теле и формулы Стирлинга для всех $\lambda$ справедлива верхняя оценка

$$
e_{L}(\lambda) \leqslant W^{1 / L} \sqrt{\frac{2 L}{\pi e}} .
$$

Таким образом, для успешного прохождения спектрального теста в сильной форме надо, чтобы нижняя граница $e_{L}(\lambda)$ отличалась от правой части (6), грубо говоря, не более, чем в 2 раза.

В данной работе определяется класс множителей $\lambda$, для которых можно написать нетривиальную оценку числа $e_{L}(\lambda)$ снизу. Заметим, что эта оценка весьма далека от того, чтобы утверждать, что множители данного класса проходят спектральный тест в сильной форме. Тем не менее, оказывается, что при специальном выборе параметров число $l$ гарантируемых множителем данного класса случайных старших $p$-ичных разрядов элементов последовательности (3) будет достаточно близко к своей верхней границе

$$
l \leqslant \frac{F}{L}+\frac{\ln \sqrt{2 L /(\pi e)}}{\ln p},
$$

вытекающей из (6). Точная формулировка дается следующей теоремой.

Теорема 1. Пусть челье числа $p, F, W=p^{F}$ и $L \geqslant 2-$ - же же, что и выше. Целые числа $E, a, T$ удовлетворяют условиям

$$
p^{E} \geqslant 3, \quad T \geqslant L, \quad p \nmid a T, \quad|a| \leqslant p^{E} .
$$

Целье числа $a^{\prime}, T^{\prime}$ удовлетворяют условиям

$$
a a^{\prime} \equiv 1 \quad(\bmod W), \quad T T^{\prime} \equiv 1 \quad(\bmod W), \quad T^{\prime}>0 .
$$

Множитель $\lambda$ имеет вид

$$
\lambda=\left(1-p^{E} a^{\prime}\right)^{T^{\prime}} .
$$


Тогда при достаточно большом $T$

$$
e_{L}(\lambda) \geqslant W^{1 / L-(1-1 / L) \delta}, \quad \delta=\frac{1}{\lceil F /(E L)\rceil-1}+\frac{5,414(L-1) \ln T}{E \ln p} .
$$

Для прояснения содержания этой теоремы сделаем некоторые замечания.

1. Числа $\lambda$ этой теоремы удовлетворяют условию (4), налагаемому на множитель для генерации последовательностей (3) периода максимальной длины.

2. Неравенство (8) становится тривиальным, если величина, стоящая в показателе степени $W$, неположительна, в частности, если

$$
\rho=\lceil F /(E L)\rceil \leqslant L
$$

или

$$
E \ln p \leqslant 5,414(L-1)^{2} \ln T .
$$

Следовательно, чтобы утверждение теоремы было содержательным, надо как минимум предполагать, что $\rho>L$ и $E \ln p>5,414(L-1)^{2} \ln T$. Отсюда, в частности, вытекает, что $F>E L^{2}$ и $p^{E}>T^{5.414(L-1)^{2}}$, то есть $W=p^{F}>T^{5.414 L^{2}(L-1)^{2}}$. Таким образом, число $W$ должно расти вместе с $T$ по меньшей мере, как $T^{5.414 L^{2}(L-1)^{2}}$.

3. При некоторых условиях можно добиться того, чтобы число $\delta$ было произвольно мало. Например, если обе величины

$$
E_{0}=\sqrt{\frac{F \ln T}{\ln p}}, \quad \rho_{0}=\frac{F}{E_{0} L}=\sqrt{\frac{F \ln p}{L^{2} \ln T}}
$$

достаточно велики, то для $E=\left\lfloor 2,327 E_{0}\right\rfloor$ получим, что $\delta<4,654 \rho_{0}$.

4. Число $l$ гарантируемых множителем $\lambda$ теоремы случайных старших $p$-ичных разрядов элементов последовательности (3) удовлетворяет неравенству

$$
l \geqslant\left\lfloor\frac{F}{L}(1-(L-1) \delta)\right\rfloor,
$$

что асимптотически равно (7), если $(L-1) \delta$ стремится к 0.

5. Неравенство (5) спектрального теста в сильной форме для чисел $\lambda$ теоремы может не выполняться, но исходя из результатов практических вычислений можно предположить, что при правильном выборе параметров это будет довольно редким явлением (см. раздел 5).

В заключение заметим, что метод доказательства теоремы целиком опирается на хорошо известную конструкцию приближений Эрмита-Паде для совокупности степенных функций, примененную к р-адическому случаю. Главная проблема при этом состоит в получении точной верхней оценки для наименьшего общего знаменателя коэффициентов этих приближений. При тривиальной оценке этой величины получается неравенство типа (8) с величиной $T$ вместо $\ln T$. Основной вклад нашей работы состоит в том, что мы значительно уточняем верхнюю оценку знаменателя, делая это применительно к нашему случаю в целом на основе тех же идей, что использованы в лемме 2.5 в [7], при доказательстве формулы 5.7 в [8], в лемме 2.5 в [9] и в предложении 6.6 в [6]. Наша основная лемма (см. ниже лемму 3) отличается от вышеперечисленных тем, что в ней рассматривается случай произвольного большого $T$ и произвольного $\rho$, что вносит ряд дополнительных трудностей в структуру доказательства. 


\section{2. Оценка $\pi(X, K, \Lambda)$}

Всюду в данной работе $q$ обозначает простое число, $q_{1}, q_{2}, \ldots$ обозначают все простые числа, занумерованные в возрастающем порядке, $c_{1}, C_{1}, c_{2}, C_{2}, \ldots$ обозначают некоторые положительные абсолютные постоянные. Знаки $O$ также скрывают некоторые положительные абсолютные постоянные.

Лемма 1. При $K \rightarrow \infty u X \geqslant 2^{(1+\varepsilon)(\ln K) / \ln \ln K}$, где $\varepsilon-$ любое положительное число, справедлива оченка

$$
\sum_{x \leqslant X,(x, K)=1} \frac{1}{x}=\frac{\varphi(K)}{K} \ln X(1+o(1)),
$$

где $\varphi(K)$ - функиия Эйлера, $a(x, K)$ - наибольший общий делитель.

Доказательство. Пусть

$$
S(X)=\sum_{x \leqslant X,(x, K)=1} \frac{1}{x}, \quad R(X)=\sum_{x=1}^{X} \frac{1}{x}
$$

и $\mu(d)$ - функщия Мебиуса. Так как $\sum_{d \mid t} \mu(d)$ есть 0 при $t>1$ и 1 при $t=1$ (см. [1]), справедливы равенства

$$
\begin{aligned}
S(X) & =\sum_{t \mid K} \sum_{x \leqslant X,(x, K)=t} \frac{1}{x} \sum_{d \mid t} \mu(d)=\sum_{d \mid K} \mu(d) \sum_{t: d|t, t| K} \sum_{x \leqslant X,(x, K)=t} \frac{1}{x} \\
& =\sum_{d \mid K} \mu(d) \sum_{x \leqslant X, d \mid x} \frac{1}{x}=\sum_{d \mid K} \mu(d) \sum_{x \leqslant X / d} \frac{1}{x d}=\sum_{d \mid K} \frac{\mu(d)}{d} R(X / d) .
\end{aligned}
$$

Так как $R(X)=\ln X+O(1)$ при $X \geqslant 1$ и 0 при $X<1$,

$$
S(X)=\sum_{d \mid K, d \leqslant X} \frac{\mu(d)}{d} \ln X-\sum_{d \mid K, d \leqslant X} \frac{\mu(d)}{d} \ln d+O\left(\sum_{d \mid K} \frac{1}{d}\right) .
$$

Сумма под знаком $O$ не превосходит

$$
\prod_{q \mid K} \sum_{k=0}^{\infty} \frac{1}{q^{k}}=\prod_{q \mid K} \frac{q}{q-1}=\frac{K}{\varphi(K)}
$$

(последнее равенство приведено в [1]), и это асимптотически малая величина по сравнению с предполагаемым главным членом $(\varphi(K) \ln X) / K$. Для доказательства заметим, чTO

$$
\frac{\varphi(K)}{K} \geqslant \frac{c_{1}}{\ln \ln K}, \quad K \geqslant 3,
$$

где $c_{1}>0$ - абсолютная постоянная (см. теорему 5.1 в [5]). Поскольку в силу условия леммы $\ln X>0,6(\ln K) / \ln \ln K$, то

$$
\frac{\varphi(K)}{K} \ln X \geqslant \frac{0,6 c_{1} \ln K}{(\ln \ln K)^{2}},
$$


в то время как

$$
\frac{K}{\varphi(K)}=O(\ln \ln K)=o\left(\frac{\ln K}{(\ln \ln K)^{2}}\right),
$$

что и требовалось доказать.

Так как

$$
\sum_{d \mid K} \frac{\mu(d)}{d}=\frac{\varphi(K)}{K}
$$

(см. [1]), соотношение (9) переписывается в виде

$$
S(X)=\frac{\varphi(K)}{K} \ln X(1+o(1))-\sum_{d \mid K, d>X} \frac{\mu(d)}{d} \ln X-\sum_{d \mid K, d \leqslant X} \frac{\mu(d)}{d} \ln d .
$$

Для доказательства леммы нам остается показать, что две последние суммы асимптотически малы по сравнению с $(\varphi(K) \ln X) / K$.

Покажем сперва, что

$$
\sum_{d \mid K} \frac{\ln d}{d} \mu(d)=O\left((\ln \ln K)^{2} \ln \ln \ln K\right) .
$$

Очевидно, что достаточно рассмотреть случай, когда $K$ не делится на квадрат простого числа. Если $K$ делится на $q^{\alpha}, \alpha>1$, то $K$ можно разделить на $q^{\alpha-1}$, при этом левая часть (13) не изменится. При этом условии докажем более сильное неравенство

$$
\sum_{d \mid K} \frac{\ln d}{d}=O\left((\ln \ln K)^{2} \ln \ln \ln K\right) .
$$

Пусть $r$ - число простых чисел, входящих в разложение $K$. Функщия $(\ln d) / d$ монотонно убывает при $d \geqslant 3$, поэтому, если мы докажем (14) для числа $K_{0}=q_{1} q_{2} \ldots q_{r}$, то это же будет верно и для $K$ ввиду того, что

$$
\sum_{d \mid K} \frac{\ln d}{d} \leqslant \frac{\ln 2}{2}+\sum_{d \mid K_{0}} \frac{\ln d}{d}
$$

Следовательно, при доказательстве (14) можно предполагать, что число $K$ равно произведению первых $r$ простых чисел. Тогда

$$
\begin{aligned}
\sum_{d \mid K} \frac{\ln d}{d} & =\sum_{s=1}^{r} \sum_{1 \leqslant k_{1}<\ldots<k_{s} \leqslant r} \frac{\ln \left(q_{k_{1}} \ldots q_{k_{s}}\right)}{q_{k_{1}} \ldots q_{k_{s}}} \leqslant \sum_{s=1}^{r} s \sum_{1 \leqslant k_{1}<\ldots<k_{s} \leqslant r} \frac{\ln q_{k_{s}}}{q_{k_{1}} \ldots q_{k_{s}}} \\
& \leqslant \sum_{s=1}^{r} \frac{s}{(s-1) !} \sum_{k_{i}<k_{s} \text { при } i<s, s \leqslant k_{s} \leqslant r} \frac{\ln q_{k_{s}}}{q_{k_{1}} \ldots q_{k_{s}}} \\
& =\sum_{s=1}^{r} \frac{s}{(s-1) !} \sum_{s \leqslant k_{s} \leqslant r} \frac{\ln q_{k_{s}}}{q_{k_{s}}}\left(\sum_{1 \leqslant k<k_{s}} \frac{1}{q_{k}}\right)^{s-1}
\end{aligned}
$$

(при $s=1, k_{s}=1$ величина $\left(\sum_{1 \leqslant k<k_{s}} 1 / q_{k}\right)^{s-1}$ считается равной 1 ). 
При $Q \geqslant 3$ справедлива оценка (см. [2])

$$
\sum_{q \leqslant Q} \frac{1}{q}=\ln \ln Q+O(1)
$$

Полагая здесь $Q=q_{k_{s}}-1$ и учитывая, что при $k \geqslant 3$

$$
\ln q_{k}=\ln k+\ln \ln k+O(1)
$$

(см. теорему 3.3 в [5]), получим, что при $k_{s} \geqslant 3$

$$
\sum_{1 \leqslant k<k_{s}} \frac{1}{q_{k}}=\ln \ln k_{s}+O(1) .
$$

Применяя (16) и (17) к (2), найдем, что

$$
\begin{aligned}
\sum_{d \mid K} \frac{\ln d}{d} \leqslant & \sum_{s=1}^{2} \frac{s}{(s-1) !} \sum_{s \leqslant k_{s} \leqslant 2} \frac{\ln q_{k_{s}}}{q_{k_{s}}}\left(\sum_{1 \leqslant k<k_{s}} \frac{1}{q_{k}}\right)^{s-1} \\
& +\sum_{s=1}^{r} \frac{s}{(s-1) !} \sum_{3 \leqslant k_{s} \leqslant r} O\left(\frac{\ln k_{s}}{k_{s} \ln k_{s}}\right)\left(\ln \ln k_{s}+C_{1}\right)^{s-1} \\
= & O\left(1+\sum_{s=1}^{r} \frac{s}{(s-1) !} \sum_{3 \leqslant k \leqslant r} \frac{\left(\ln \ln k+C_{1}\right)^{s-1}}{k}\right) .
\end{aligned}
$$

При любом фиксированном $s \in[1, r]$ функщия $x \mapsto\left(\ln \ln x+C_{1}\right)^{s-1} / x$ имеет на отрезке $[3, r]$ не более двух участков монотонности, а значения этой функции не превосходят $\left(\ln s+C_{1}\right)^{s-1}$. Следовательно,

$$
\sum_{d \mid K} \frac{\ln d}{d}=O\left(1+\sum_{s=1}^{r} \frac{s}{(s-1) !}\left(\ln s+C_{1}\right)^{s-1}+\sum_{s=1}^{r} \frac{s}{(s-1) !} \int_{3}^{r} \frac{\left(\ln \ln x+C_{1}\right)^{s-1}}{x} d x\right) .
$$

Ряд

сходится, поэтому

$$
\sum_{s=1}^{\infty} \frac{s}{(s-1) !}\left(\ln s+C_{1}\right)^{s-1}
$$

$$
\sum_{d \mid K} \frac{\ln d}{d}=O\left(1+\sum_{s=1}^{r} \frac{s}{(s-1) !} \int_{3}^{r} \frac{\left(\ln \ln x+C_{1}\right)^{s-1}}{x} d x\right) .
$$

Далее заметим, что

поэтому

$$
\frac{\left(\ln \ln x+C_{1}\right)^{s-1}}{x} \leqslant \frac{d}{d x}\left(\left(\ln \ln x+C_{1}\right)^{s-1} \ln x\right),
$$

$$
\begin{aligned}
\sum_{d \mid K} \frac{\ln d}{d} & =O\left(1+\left.\sum_{s=1}^{r} \frac{s}{(s-1) !}\left(\left(\ln \ln x+C_{1}\right)^{s-1} \ln x\right)\right|_{3} ^{r}\right) \\
& =O\left(1+\sum_{s=1}^{r} \frac{s}{(s-1) !}\left(\ln \ln r+C_{1}\right)^{s-1} \ln r\right)
\end{aligned}
$$


Так как

$$
\sum_{s=1}^{\infty} \frac{s x^{s-1}}{(s-1) !}=(x+1) e^{x}
$$

To

$$
\sum_{d \mid K} \frac{\ln d}{d}=O\left(1+\ln r\left(\ln \ln r+C_{1}+1\right) \ln r\right)=O\left(\ln ^{2} r \ln \ln r\right) .
$$

С другой стороны, ясно, что $r=O(\ln K)$, откуда,

$$
\ln r=O(\ln \ln K), \quad \ln \ln r=O(\ln \ln \ln K)
$$

и оценка (13) тем самым доказана.

Заметим, что правая часть (13) асимптотически мала по сравнению с предполагаемым главным членом $(\varphi(K) / K) \ln X$ (см. (11)), поэтому из (12) и (13) следует, что

$$
S(X)=\frac{\varphi(K)}{K} \ln X(1+o(1))+\sum_{d \mid K, d>X} \frac{\mu(d)}{d} \ln \frac{d}{X} .
$$

Отсюда вытекает, что

$$
S(X)=\frac{\varphi(K)}{K} \ln X(1+o(1))+O\left(\frac{\ln K}{X}\right) \sum_{d \mid K} 1 .
$$

По теореме 5.2 из [5] сумма $\sum_{d \mid K} 1$ при достаточно большом $K$ не превосходит

$$
\exp \left((1+\varepsilon / 2) \frac{\ln K}{\ln \ln K} \ln 2\right)
$$

значит,

$$
S(X)=\frac{\varphi(K)}{K} \ln X(1+o(1))+O\left(\frac{\ln K}{X} \exp \left((1+\varepsilon / 2) \frac{\ln K}{\ln \ln K} \ln 2\right)\right) .
$$

Вспоминая, что по условию леммы число $X$ не меньше, чем

$$
\exp \left((1+\varepsilon) \frac{\ln K}{\ln \ln K} \ln 2\right)
$$

получим, что

$$
S(X)=\frac{\varphi(K)}{K} \ln X(1+o(1))+O\left(\exp \left(-\frac{\varepsilon}{2} \frac{\ln K}{\ln \ln K} \ln 2\right)\right) .
$$

Остаточный член в этой формуле асимптотически мал по сравнению с $(\varphi(K) / K) \ln X$ (что следует из (11)), значит,

$$
S(X)=\frac{\varphi(K)}{K} \ln X(1+o(1))
$$

и лемма тем самым доказана.

Ниже мы используем стандартное обозначение $\pi(X, K, \Lambda)$ числа простых чисел, не превосходящих $X$ и сравнимых с $\Lambda$ по модулю $K$ (предполагается, что $1 \leqslant \Lambda \leqslant K$ и наибольший общий делитель $(\Lambda, K)=1)$. 
Лемма 2. При $K \rightarrow \infty u X \geqslant K 4^{(1+\varepsilon) \ln K / \ln \ln K}$, где $\varepsilon-$ произвольное положительное число, справедлива оченка

$$
\pi(X, K, \Lambda) \leqslant \frac{2+o(1)}{\varphi(K)} \frac{X}{\ln (X / K)} .
$$

Доказательство. При доказательстве теоремы 4.1 в главе 2 в [5] было установлено неравенство

$$
\pi(X, K, \Lambda) \leqslant \frac{N}{Z}+R+\frac{z}{K}+1
$$

где

$$
\begin{array}{lll}
N=|\{m \in \mathbf{N}: K m+\Lambda \leqslant X\}|=\frac{X}{K}+O(1), & z=\sqrt{N} / \ln ^{2} N, \\
Z=\sum_{m \leqslant z,(m, K)=1} \frac{1}{m}, & R=O\left(z^{2} \ln ^{2} z\right) .
\end{array}
$$

Отметим, что аналогичное неравенство содержится в [5], однако в [5] дается недостаточно точная для наших целей оценка числа $Z$ снизу, что ведет к недостаточно точной верхней границе для $\pi(X, K, \Lambda)$. Цель данной леммы состоит в том, чтобы подправить оценку для $Z$ (пользуясь леммой 1 ) и получить таким образом требуемую оценку для $\pi(X, K, \Lambda)$.

Чтобы использовать неравенство

$$
Z \geqslant \frac{\varphi(K)}{K} \ln z(1+o(1))
$$

леммы 1 , мы должны проверить, что выполнено требуемое ею условие для числа $z$, то есть, что

$$
z \geqslant 2^{(1+\varepsilon / 2)(\ln K) / \ln \ln K}
$$

Заметим, что из определения числа $N$ и условия на $X$ следует, что

$$
N \geqslant \frac{X}{K}-2 \geqslant 4^{(1+\varepsilon)(\ln K) / \ln \ln K}-2 \text {. }
$$

Так как $z=\sqrt{N} / \ln ^{2} N$ и функция $\sqrt{x} / \ln ^{2} x$ монотонно растет при $x \geqslant e^{4}$, для доказательства (20) достаточно показать, что

$$
\frac{\sqrt{4^{(1+\varepsilon)(\ln K) / \ln \ln K}-2}}{\ln ^{2}\left(4^{(1+\varepsilon)(\ln K) / \ln \ln K}-2\right)} \geqslant 2^{(1+\varepsilon / 2)(\ln K) / \ln \ln K} .
$$

Последнее неравенство при достаточно большом $K$, очевидно, выполнено. Используя (18) и (19) и учитывая, что $R=O\left(z^{2} \ln ^{2} z\right)=O\left(N / \ln ^{2} N\right)$, приходим к оценке

$$
\begin{aligned}
\pi(X, K, \Lambda) & \leqslant \frac{N K}{\varphi(K)} \frac{1+o(1)}{\ln z}+O\left(\frac{N}{\ln ^{2} N}\right) \\
& =\frac{2 N K(1+o(1))}{\varphi(K) \ln N}+O\left(\frac{N}{\ln ^{2} N}\right) .
\end{aligned}
$$


Так как $K$ больше, чем $\varphi(K)$, второе слагаемое правой части (21) асимптотически мало по сравнению с первым. Учитывая также, что

$$
N=\frac{X}{K}+O(1)=\frac{X}{K}(1+o(1)),
$$

и следовательно,

$$
\ln N=\ln \frac{X}{K}+o(1)=(1+o(1)) \ln \frac{X}{K}
$$

из (21) найдем, что

$$
\pi(X, K, \Lambda) \leqslant \frac{2+o(1)}{\varphi(K)} \frac{X}{\ln (X / K)},
$$

что и требовалось доказать.

\section{3. Лемма о знаменателе}

Для формулировки следующей леммы введем обозначения

$$
\begin{aligned}
(x ; T)_{a}^{A} & =(x+a T)(x+(a+1) T) \ldots(x+(A-1) T), \\
(x)_{a}^{A} & =(x ; 1)_{a}^{A} .
\end{aligned}
$$

Пусть также $\operatorname{den} X$ обозначает совокупность знаменателей множества $X \subseteq \mathbf{Q}$, то есть множество таких $D \in \mathbf{Q}$, что $D x \in \mathbf{Z}$ для любого $x \in X$.

Лемма 3. Пусть

$$
\begin{gathered}
m \in \mathbf{Z}_{\geqslant 1}, \quad \xi_{1}, \ldots, \xi_{m} \in \mathbf{Q}, \quad n \in \mathbf{Z}_{\geqslant 1}, \quad \eta_{1}, \ldots, \eta_{n} \in \mathbf{Q} ; \\
0<\xi_{i} \leqslant 1, \quad 0<\eta_{j} \leqslant 1, \quad i=1, \ldots, m, \quad j=1, \ldots, n ; \\
T \in \operatorname{den}\left\{\xi_{i}, \eta_{j}: i=1, \ldots, m, \quad j=1, \ldots, n\right\} \cap \mathbf{Z}_{\geqslant 2}, \quad R \in \mathbf{Z}_{\geqslant 2} ;
\end{gathered}
$$

$\Theta-$ множество чисел $\theta$ вида

$$
\prod_{i \in\left\{i_{1}, \ldots, i_{M}\right\}}\left(\xi_{i}\right)_{a_{i}}^{A_{i}} / \prod_{j \in\left\{j_{1}, \ldots, j_{N}\right\}}\left(\eta_{j}\right)_{b_{j}}^{B_{j}},
$$

где

$$
\begin{array}{rrr}
M \in \mathbf{Z}_{\geqslant 1}, & i_{1}, \ldots, i_{M} & - \text { произвольные числа из }\{1, \ldots, m\} ; \\
a_{i}, A_{i} \in \mathbf{Z}, & 0 \leqslant a_{i}<A_{i}<R, & i=i_{1}, \ldots, i_{M} ; \\
N \in \mathbf{Z}_{\geqslant 1}, & j_{1}, \ldots, j_{N} & - \text { попарно различные числа из }\{1, \ldots, n\} ; \\
b_{j}, B_{j} \in \mathbf{Z}, & 0 \leqslant b_{j}<B_{j}<R, & j=j_{1}, \ldots, j_{N} ; \\
& \sum_{i \in\left\{i_{1}, \ldots, i_{M}\right\}}\left(A_{i}-a_{i}\right)=\sum_{j \in\left\{j_{1}, \ldots, j_{N}\right\}}\left(B_{j}-b_{j}\right) .
\end{array}
$$

Тогда справедливы следующие утверждения.

(1) $\ln \theta \leqslant R n+n \ln (R T)$ для всех $\theta \in \Theta$;

(2) При достаточно большом $T$ и $M=O(n)$ существует положительное число $D \in \operatorname{den} \Theta$, удовлетворяющее неравенству $\ln D \leqslant 3,86036 R n \ln T$. 
(3) Если в условиях леммы все $\xi_{1}, \ldots, \xi_{m}$ равньі 1 или для всех $d \mid T$

$$
\left|\left\{j=\{1, \ldots, n\}: d \mid T \eta_{j}\right\}\right| \leqslant \frac{n}{d},
$$

то константу 3,86036 можно заменить на 3,207.

Прежде, чем перейти к доказательству, заметим, что число $T$ в данной лемме не обязано совпадать с числом $T$ в сформулированной выше теореме, хотя, как мы увидим далее, при доказательстве теоремы реализуется именно этот случай.

Доказательство. Докажем утверждение 1. Пусть

$$
\begin{aligned}
& \theta=\prod_{i \in\left\{i_{1}, \ldots, i_{M}\right\}}\left(\xi_{i}\right)_{a_{i}}^{A_{i}} / \prod_{j \in\left\{j_{1}, \ldots, j_{N}\right\}}\left(\eta_{j}\right)_{b_{j}}^{B_{j}}, \\
& S=\sum_{i \in\left\{i_{1}, \ldots, i_{M}\right\}}\left(A_{i}-a_{i}\right)=\sum_{j \in\left\{j_{1}, \ldots, j_{N}\right\}}^{N}\left(B_{j}-b_{j}\right) .
\end{aligned}
$$

Так как $A_{i}-a_{i} \leqslant R-1$ и $\xi_{i} \leqslant 1$, то $\left(\xi_{i}\right)_{a_{i}}^{A_{i}} \leqslant(1+R-1)^{A_{i}-a_{i}}$ и

$$
\ln \prod_{i=i_{1} \ldots \ldots, i_{M}}\left(\xi_{i}\right)_{a_{i}}^{A_{i}} \leqslant S \ln R
$$

С другой стороны, $\eta_{j} \geqslant 1 / T$, поэтому

$$
\begin{aligned}
\left(\eta_{j}\right)_{b_{j}}^{B_{j}} & \geqslant \frac{1}{T}\left(\frac{1}{T}+1\right) \ldots\left(\frac{1}{T}+B_{j}-b_{j}-1\right) \\
& \geqslant \frac{1}{T}\left(B_{j}-b_{j}-1\right) !
\end{aligned}
$$

Пусть

$$
S_{0}=\sum_{j \in\left\{j_{1}, \ldots, j_{N}\right\}}\left(B_{j}-b_{j}-1\right)=S-N
$$

Тогда в силу (24)

$$
\ln \prod_{j \in\left\{j_{1}, \ldots, j_{N}\right\}}\left(\eta_{j}\right)_{b_{j}}^{B_{j}} \geqslant-n \ln T+\ln \left(S_{0} !\right)-\ln \frac{\left(B_{j_{1}}-b_{j_{1}}-1+\ldots+B_{j_{N}}-b_{j_{N}}-1\right) !}{\left(B_{j_{1}}-b_{j_{1}}-1\right) ! \cdot \ldots \cdot\left(B_{j_{N}}-b_{j_{N}}-1\right) !}
$$

Последнее слагаемое правой части этого неравенства есть минус логарифм от коэффициента при члене $X_{1}^{B_{j_{1}}-b_{j_{1}}-1} \ldots X_{N}^{B_{j_{N}}-b_{j_{N}}-1}$ в разложении степени $\left(X_{1}+\ldots+X_{N}\right)^{S_{0}}$ в сумму одночленов от $X_{1}, \ldots, X_{N}$. Этот коэффициент в любом случае не превосходит $(1+\ldots+1)^{S_{0}}=N^{S_{0}}$, следовательно,

$$
\ln \prod_{j \in\left\{j_{1} \ldots, j_{N}\right\}}\left(\eta_{j}\right)_{b_{j}}^{B_{j}} \geqslant-n \ln T+\ln \left(S_{0} !\right)-S_{0} \ln N .
$$


Используя формулу Стирлинга и учитывая, что $N \leqslant n$ (что следует из условия леммы), получим, что

$$
\begin{aligned}
\ln \prod_{j \in\left\{j_{1} \ldots, j_{N}\right\}}\left(\eta_{j}\right)_{b_{j}}^{B_{j}} & \geqslant-n \ln T+S_{0} \ln \frac{S_{0}}{e}-S_{0} \ln N \\
& \geqslant-n \ln T+S_{0} \ln S_{0}-S_{0} \ln (n e),
\end{aligned}
$$

отсюда, ввиду (23) и (25), для числа $\theta$ из (22) получаем оценку

$$
\ln \theta \leqslant S_{0} \ln (n e R)+n \ln R+n \ln T-S_{0} \ln S_{0} .
$$

Далее заметим, что функция $x \mapsto x \ln (n e R)-x \ln x$ монотонно растет при $0 \leqslant x \leqslant n R$. Так как $S_{0}<S<n R$ (второе неравенство вытекает из того, что $A_{i}-a_{i} \leqslant R-1$ и $N \leqslant n$ ), из (26) следует оценка

$$
\ln \theta \leqslant n R \ln (n e R)+n \ln (R T)-n R \ln (n R)=R n+n \ln (R T),
$$

что и требовалось доказать.

Докажем утверждение 2. Пусть $\xi_{i}=x_{i} . T, \eta_{j}=y_{j} . T$, где $x_{i}, y_{j} \in \mathbf{Z}_{\geqslant 1}$. Запишем число $\theta$ в виде $\theta_{0} \theta_{1} \theta_{2}$, где

$$
\begin{aligned}
& \theta_{0}=\prod_{j \in\left\{j_{1}, \ldots, j_{N}\right\}} \frac{\left(B_{j}-b_{j}\right) !}{\left(y_{j} ; T\right)_{b_{j}}^{B_{j}}}, \\
& \theta_{1}=\prod_{i \in\left\{i_{1}, \ldots, i_{M}\right\}} \frac{\left(x_{i} ; T\right)_{a_{i}}^{A_{i}}}{\left(A_{i}-a_{i}\right) !} \\
& \theta_{2}=\prod_{i \in\left\{i_{1} \ldots ., i_{M}\right\}}\left(A_{i}-a_{i}\right) ! / \prod_{j \in\left\{j_{1}, \ldots, j_{N}\right\}}\left(B_{j}-b_{j}\right) !
\end{aligned}
$$

Пусть $\Theta_{\sigma}-$ множества чисел вида $\theta_{\sigma}$ для $\sigma=0,1,2$, удовлетворяющих условиям леммы, налагаемым на множество $\Theta$. Мы покажем, что существуют элементы $0<D_{\sigma} \in \operatorname{den} \Theta_{\sigma}$ такие, что

$$
\begin{aligned}
& \ln D_{0} \leqslant 3,860357 R n \ln T, \\
& \ln D_{1}=O(R n \ln \ln T), \\
& \ln D_{2}=O(R n) .
\end{aligned}
$$

Этого будет достаточно для доказательства утверждения 2.

Пусть сначала $\sigma=2$. Если $q$ - простое число, $q \leqslant \rho$, и $v_{q}(x)-q$-показатель числа $x$ (то есть наибольшее целое $\alpha$, для которого $q^{\alpha} \mid x$ ), то

$$
v_{q}\left(\theta_{2}\right)=\sum_{\alpha=1}^{\infty}\left(\sum_{i \in\left\{i_{1}, \ldots, i_{M}\right\}}\left\lfloor\left(A_{i}-a_{i}\right) / q^{\alpha}\right\rfloor-\sum_{j \in\left\{j_{1}, \ldots, j_{N}\right\}}\left\lfloor\left(B_{j}-b_{j}\right) / q^{\alpha}\right\rfloor\right)
$$

это вытекает из известной формулы для $v_{q}(X !)$ (см. [1]):

$$
v_{q}(X !)=\sum_{\alpha=1}^{\infty}\left\lfloor X / q^{\alpha}\right\rfloor, \quad X \in \mathbf{N} .
$$


Так как $A_{i}-a_{i}<R, B_{j}-b_{j}<R$ и

$$
\sum_{i \in\left\{i_{1}, \ldots, i_{M}\right\}}\left(A_{i}-a_{i}\right)=\sum_{j \in\left\{j_{1}, \ldots, j_{N}\right\}}\left(B_{j}-b_{j}\right)
$$

из (30) следует, что

$$
\begin{aligned}
v_{q}\left(\theta_{2}\right) & \geqslant \sum_{\alpha: q^{\alpha} \leqslant R}\left(\sum_{i \in\left\{i_{1}, \ldots, i_{M}\right\}}\left(\frac{A_{i}-a_{i}}{q^{\alpha}}-1\right)-\sum_{j \in\left\{j_{1}, \ldots, j_{N}\right\}} \frac{B_{j}-b_{j}}{q^{\alpha}}\right) \\
& =-M \sum_{\alpha: q^{\alpha} \leqslant R} 1=-M\lfloor(\ln R) / \ln q\rfloor .
\end{aligned}
$$

По условию леммы $M \leqslant C_{2} n$, причем число $C_{2}$ можно считать целым, поэтому, полагая

$$
D_{2}=\prod_{q \leqslant R} q^{C_{2} n\lfloor(\ln R) / \ln q\rfloor}
$$

получим, что $0<D_{2} \in \operatorname{den} \Theta_{2}$ и

$$
\ln D_{2} \leqslant C_{2} n \sum_{q \leqslant R}\left\lfloor\frac{\ln R}{\ln q}\right\rfloor \ln q \leqslant C_{2} n \ln R \sum_{q \leqslant R} 1=O(n R),
$$

последнее соотношение справедливо в силу известной оценки Чебышева для числа простых чисел, не превосходящих $R$ (см. теорему 3.2 в [5]). Тем самым, неравенство (29) доказано.

В случаях $\sigma=1$ и $\sigma=0$ надо оценить числа $v_{q}\left(\left(x_{i} ; T\right)_{a_{i}}^{A_{i}}\right)$ снизу, а $v_{q}\left(\left(y_{j} ; T\right)_{b_{j}}^{B_{j}}\right)$ сверху. Мы будем использовать следующее двустороннее неравенство: если $y, b$ и $\boldsymbol{B}$ целые числа, лежащие в пределах $1 \leqslant y \leqslant T, 0 \leqslant b<B<R$, и $q$ - простое число, не делящее $T$, то

$$
v_{q}((B-b) !) \leqslant v_{q}(y ; T)_{b}^{B} \leqslant v_{q}((B-b) !)+\left\lfloor\frac{\ln (R T)}{\ln q}\right\rfloor
$$

(см. доказательство теоремы 4.3 в [6], именно, оценку числа $\Delta_{r}^{(1)}$ ).

Рассмотрим случай $\sigma=1$. Пусть $q$ - простое число. Тогда, если $q \nmid T$, то справедливо неравенство $v_{q}\left(\theta_{1}\right) \geqslant 0$. Если же $q \mid T$, то во всяком случае

$$
\begin{aligned}
v_{q}\left(\theta_{1}\right) & \geqslant v_{q}\left(\prod_{i \in\left\{i_{1} \ldots \ldots, i_{M}\right\}} \frac{1}{\left(A_{i}-a_{i}\right) !}\right)=-\sum_{i \in\left\{i_{1}, \ldots, i_{M}\right\}} \sum_{\alpha=1}^{\infty}\left\lfloor\frac{A_{i}-a_{i}}{q^{\alpha}}\right\rfloor \\
& >-\sum_{i \in\left\{i_{1} \ldots, i_{M}\right\}} \sum_{\alpha=1}^{\infty} \frac{A_{i}-a_{i}}{q^{\alpha}}=-\frac{S}{q-1}>-\frac{R n}{q-1}
\end{aligned}
$$

(то, что $S<R n$, мы уже видели при доказательстве утверждения 1).

Положим

$$
D_{1}=\prod_{q \mid T} q^{\lfloor R n /(q-1)\rfloor} .
$$


Тогда $0<D_{1} \in \operatorname{den} \Theta_{1}$ и

$$
\ln D_{1} \leqslant R n \sum_{q \mid T} \frac{\ln q}{q-1} .
$$

Для доказательства (28) нам остается показать, что

$$
\sum_{q \mid T} \frac{\ln q}{q-1}=O(\ln \ln T) .
$$

Пусть $r$ - число простых чисел, входящих в разложение $T$. Так как функщия $(\ln x) /(x-1)$ при $x \geqslant 2$ монотонно убывает, не ограничивая общности, можно считать, что $T=q_{1} q_{2} \ldots q_{r}$ (рассуждение здесь то же, что и при доказательстве неравенства (14) леммы 1). Пользуясь оценкой (16), получаем, что

$$
\begin{aligned}
\sum_{q \mid T} \frac{\ln q}{q-1} & =\sum_{k=1}^{2} \frac{\ln q_{k}}{q_{k}-1}+\sum_{k=3}^{r} \frac{\ln q_{k}}{q_{k}-1} \\
& =O\left(1+\sum_{k=3}^{r} \frac{\ln k}{k \ln k}\right)=O(\ln r) .
\end{aligned}
$$

Так как $r=O(\ln T)$, то $\ln r=O(\ln \ln T)$, что и доказывает (33). Этим завершается рассмотрение случая $\sigma=1$.

Пусть теперь $\sigma=0$. Рассмотрим два случая.

1. Пусть $R \leqslant T^{\alpha}$, где $\alpha \in(1,1,2,860357)$ - параметр, который мы уточним ниже. Положим

$$
D_{0}=\prod_{j=1}^{n} \prod_{h=0}^{R-1}\left(y_{j}+T h\right) .
$$

Ясно, что $0<D_{0} \in \operatorname{den} \Theta_{0}$ и

$$
\ln D_{0} \leqslant R n \ln (T R) \leqslant(\alpha+1) R n \ln T<3,860357 R n \ln T,
$$

что и требовалось в (27).

2. Пусть $R>T^{\alpha}$. Число $D_{0}$ будем искать в виде $D_{0}=D_{0,0} D_{0,1} D_{0,2}$, где $D_{0, k}>0$, $k=0,1,2$, будут определены позднее. Число $D_{0,0}$ будет отвечать за простые $q \mid T$, число $D_{0,1}$ - за простые $q \nmid T, q \leqslant R$, число $D_{0,2}$ - за остальные простые $q$. Тогда для доказательства (27) достаточно показать, что

$$
\begin{aligned}
& \ln D_{0,0} \leqslant(1+o(1)) R n \ln T, \\
& \ln D_{0,1}=O(R n), \\
& \ln D_{0,2} \leqslant(\alpha+o(1)) R n \ln T .
\end{aligned}
$$

Пусть $q$ - простое число, $q \mid T$. Оценим кратность вхождения таких $q$ в знаменатели чисел $\theta_{0} \in \Theta_{0}$. Введем обозначения $u=v_{q}(T)$ и

$$
\pi_{j}=\left(y_{j} ; T\right)_{b_{j}}^{B_{j}} /\left(B_{j}-b_{j}\right) !, \quad j=1, \ldots, n .
$$


Фиксируем временно число $j$. Пусть $v=v_{q}\left(y_{j}\right)$. Тогда, если $v<u$, то

$$
v_{q}\left(\pi_{j}\right)=v\left(B_{j}-b_{j}\right)-v_{q}\left(\left(B_{j}-b_{j}\right) !\right) \leqslant v R .
$$

Если же $v \geqslant u$, то, пользуясь (31), получим, что

$$
v_{q}\left(\pi_{j}\right) \leqslant u R+\left\lfloor\frac{\ln (R T)}{\ln q}\right\rfloor .
$$

Пусть

$$
D_{0,0}=\prod_{\substack{q \mid T \\ u=v_{q}(T)}} \prod_{\substack{1 \leqslant j \leqslant n \\ 1 \leqslant v=v_{q}\left(y_{j}\right)<u}} q^{R v} \prod_{\substack{1 \leqslant j \leqslant n \\ v=v_{q}\left(y_{j}\right) \geqslant u}} q^{\lfloor\ln (R T) / \ln q\rfloor+R u}
$$

Для всех простых $q \mid T$ и всех $\theta_{0} \in \Theta_{0}$ справедливы неравенства $v_{q}\left(D_{0,0} \theta_{0}\right) \geqslant 0$ и

$$
\begin{aligned}
\ln D_{0.0} & \leqslant \sum_{\substack{q \mid T \\
u=v_{q}(T)}}\left(R n u+n\left\lfloor\frac{\ln (R T)}{\ln q}\right\rfloor\right) \ln q \\
& \leqslant R n \ln T+n \ln (R T) \sum_{q \mid T} 1 .
\end{aligned}
$$

Так как $R>T^{\alpha}>T$, то

$$
\ln (R T)=O(\ln R)=o(R)
$$

Ясно также, что

$$
\sum_{q \mid T} 1=O(\ln T)
$$

Тем самым, установлена оценка (35) и рассмотрение случая $k=0$ завершается.

Пусть $k=1$, то есть рассматриваются простые $q \leqslant R$, не делящие $T$. Для таких $q$ в силу (31) справедливы оценки

$$
\nu_{q}\left(\pi_{j}\right) \leqslant\left\lfloor\frac{\ln (R T)}{\ln q}\right\rfloor, \quad j=1, \ldots, n .
$$

Возьмем

$$
D_{0,1}=\prod_{\substack{q \nmid T \\ q \leqslant R}} q^{n\lfloor(\ln (R T)) / \ln q\rfloor} .
$$

Тогда $v_{q}\left(D_{0,1} \theta_{0}\right) \geqslant 0$ для всех рассматриваемых $q$ и всех $\theta_{0} \in \Theta_{0}$. Пользуясь тем, что $\ln (R T)=O(\ln R)$ (так как $R>T$ ) и неравенством Чебышева для числа простых, не превосходящих $R$ (см. теорему 3.2 в [5]), находим, что

$$
\ln D_{0,1} \leqslant n \ln (R T) \sum_{\substack{q \nmid T \\ q \leqslant R}} 1=O\left(n \ln R \sum_{q \leqslant R} 1\right)=O(R n) .
$$


Тем самым, установлена оценка (36) и рассмотрение случая $k=1$ завершено.

Пусть $q$ - простое число, $q>R$. Оценим кратность вхождения таких $q$ в знаменатели чисел $\theta_{0} \in \Theta_{0}$. Прежде всего заметим, что $q \nmid\left(B_{j}-b_{j}\right)$ ! (так как $B_{j}-b_{j}<R$ ), поэтому, $v_{q}\left(\pi_{j}\right)=v_{q}\left(\left(y_{j} ; T\right)_{b_{j}}^{B_{j}}\right)$.

Допустим, что $q \mid\left(y_{j} ; T\right)_{b_{j}}^{B_{j}}$ при некотором $j$. Так как $q>R$, а $y_{j}+T B_{j} \leqslant R T$, существует равенство вида

$$
A q=y_{j}+T h, \quad 0 \leqslant h<R, \quad 1 \leqslant A \leqslant T .
$$

Число $A$ не может делиться на $q$, так как $q>R>T$.

Пусть

$$
D_{0,2}=\prod_{j=1}^{n} \prod_{A=1}^{T} \prod_{\substack{R<q<R T / A \\ T \mid\left(A q-y_{j}\right)}} q
$$

Тогда $v_{q}\left(D_{0,2} \theta_{0}\right) \geqslant 0$ для всех рассматриваемых $q$ и всех $\theta_{0} \in \Theta_{0}$.

Заметим также, что если $T \mid\left(A q-y_{j}\right), d=\left(y_{j}, T\right), y_{j}=d y_{j}^{*}, T=d T^{*}$, то $d \mid A$, $A=d A^{*}, T^{*} \mid\left(A^{*} q-y_{j}^{*}\right)$ и $\left(A^{*}, T^{*}\right)=1$. Отсюда вытекает, что

$$
\begin{aligned}
& \ln D_{0,2} \leqslant \sum_{\substack{1 \leqslant j \leqslant n \\
d=\left(y_{j}, T\right) \\
y_{j}^{*}=y_{j} / d \\
T^{*}=T / d}} \sum_{\substack{1 \leqslant A^{*} \leqslant T^{*} \\
\left(A^{*} . T^{*}\right)=1}} \sum_{\substack{q \leqslant R T^{*} / A^{*} \\
q \equiv y_{j}^{*} A^{*-1}}} \ln q \\
&=\sum_{\substack{1 \leqslant j \leqslant n \\
\left(\bmod T^{*}\right) \\
d=\left(y_{j}, T\right) y_{j}^{*}=y_{j} / d \\
T^{*}=T / d}} \sum_{\substack{A^{*}=1, \ldots . T^{*} \\
\left(A^{*} . T^{*}\right)=1}} \theta\left(\frac{R T^{*}}{A^{*}}, T^{*}, y_{j}^{*} A^{*-1}\right),
\end{aligned}
$$

где

$$
\theta(X, K, \Lambda)=\sum_{\substack{q-\text { простое } \\ q \leqslant X, q=\Lambda(\bmod K)}} \ln q .
$$

Для оценки числа $\theta\left(R T^{*} / A^{*}, T^{*}, y_{j}^{*} A^{*-1}\right)$ мы рассмотрим два случая.

Если

$$
T^{*} \leqslant \exp \left(c_{2} \frac{\ln R}{\ln \ln R}\right),
$$

где $c_{2}>0$ - некоторая достаточно малая абсолютная постоянная, то по теореме 2.1 главы 9 книги [5] получим, что

$$
\theta\left(R T^{*} / A^{*}, T^{*}, y_{j}^{*} A^{*-1}\right) \leqslant \frac{(2+o(1)) R T^{*}}{\varphi\left(T^{*}\right) A^{*}} .
$$

Если

$$
T^{*} \geqslant \exp \left(c_{2} \frac{\ln R}{\ln \ln R}\right)
$$

то $T^{*}$ растет с ростом $T$ и

$$
\frac{R T^{*}}{A^{*}} \geqslant R>T^{\alpha} \geqslant T^{* \alpha}>T^{* 1.1}>T^{*} e^{\left(2 \ln T^{*}\right) / \ln \ln T^{*}}
$$


при достаточно большом $T$, следовательно, выполнены условия леммы 2. С помощью этой леммы выводим, что

$$
\begin{aligned}
\theta\left(R T^{*} / A^{*}, T^{*}, y_{j}^{*} A^{*-1}\right) & \leqslant\left(\ln \frac{R T^{*}}{A^{*}}\right) \pi\left(R T^{*} / A^{*}, T^{*}, y_{j}^{*} A^{*-1}\right) \\
& \leqslant\left(\ln \frac{R T^{*}}{A^{*}}\right) \frac{2+o(1)}{\varphi\left(T^{*}\right)} \frac{R T^{*}}{A^{*}} \frac{1}{\ln \left(R / A^{*}\right)} \\
& =\frac{(2+o(1)) R T^{*}}{\varphi\left(T^{*}\right) A^{*}} \frac{1}{1-\left(\ln T^{*}\right) / \ln \left(R T^{*} / A^{*}\right)} .
\end{aligned}
$$

В любом случае

$$
\ln D_{0,2} \leqslant R \sum_{\substack{1 \leqslant j \leqslant n, d=\left(y_{j}, T\right) \\ y_{j}^{*}=y_{j} / d, T^{*}=T / d}} \frac{T^{*}}{\varphi\left(T^{*}\right)} \sum_{\substack{1 \leqslant A^{*} \leqslant T^{*} \\\left(A^{*}, T^{*}\right)=1}} \frac{2+o(1)}{A^{*}\left(1-\left(\ln T^{*}\right) / \ln \left(R T^{*} / A^{*}\right)\right)} .
$$

Для оценки суммы по $A^{*}$ мы рассмотрим два случая.

Если $T^{*} \leqslant \ln T$, то сумму мы оценим тривиально величиной $O\left(\ln \left(T^{*}+2\right)\right)$ (заметим, что множитель $1 /\left(1-\left(\ln T^{*}\right) / \ln \left(R T^{*} / A^{*}\right)\right.$ уйдет в $O(1)$, потому что $\left.R>T^{* 1.1}\right)$, что в силу (10) дает общий вклад в оценку $D_{0,2}$, не больший

$$
O(R) \sum_{\substack{1 \leqslant j \leqslant n, d=\left(y_{j}, T\right) \\ y_{j}^{*}=y_{j} / d, T^{*}=T / d}} O\left(\ln \ln \left(T^{*}+2\right) \ln \left(T^{*}+2\right)\right)=O(R n(\ln \ln T) \ln \ln \ln T)=o(R n \ln T) .
$$

Если $T^{*} \geqslant \ln T$, то $T^{*}$ растет с ростом $T$ и мы можем применить лемму 1 . Интервал суммирования $1 \leqslant A^{*} \leqslant T^{*}$ предварительно разобьем на несколько интервалов:

$$
A^{*} \leqslant e^{S_{1}}, \quad A^{*} \in \bigcup_{S_{1}<s \leqslant S_{2}}\left(e^{s-1} ; e^{s}\right],
$$

где

$$
S_{1}=\left\lceil\left(\ln T^{*}\right) / \ln \ln T^{*}\right\rceil, \quad S_{2}=\left\lceil\ln T^{*}\right\rceil .
$$

Строго говоря, множество целых чисел промежутка $\left(e^{S_{1}} ; T^{*}\right]$ может не совпадать с множеством целых чисел промежутка

$$
\left(e^{S_{1}} ; e^{S_{2}}\right]=\bigcup_{S_{1}<s \leqslant S_{2}}\left(e^{s-1} ; e^{s}\right],
$$

но нам достаточно того, что первое из этих множеств лежит во втором.

Сумма по $A^{*} \leqslant e^{S_{1}}$ в силу леммы 1 есть $O\left(\left(\varphi\left(T^{*}\right) \ln T^{*}\right) / T^{*} \ln \ln T^{*}\right)$ и ее вклад в оценку числа $D_{0,2}$ есть $O((R n \ln T) / \ln \ln T)=o(R n \ln T)$.

На промежутке $e^{s-1}<A^{*} \leqslant e^{s}$ справедлива оценка сверху

$$
\begin{aligned}
\frac{1}{1-\left(\ln T^{*}\right) / \ln \left(R T^{*} / A^{*}\right)} & \leqslant \frac{1}{1-\left(\ln T^{*}\right) / \ln \left(R T^{*} / e^{s}\right)} \\
& <\frac{1}{1-\left(\ln T^{*}\right) / \ln \left(T^{*}(\alpha+1) / e^{s}\right)} \\
& =\frac{(\alpha+1) \ln T^{*}-s}{\alpha \ln T^{*}-s} \\
& =1+\frac{\ln T^{*}}{\alpha \ln T^{*}-s},
\end{aligned}
$$


поэтому

$$
\sum_{\substack{e^{s-1}<A^{*} \leqslant e^{s} \\\left(A^{*}, T^{*}\right)=1}} \frac{1}{A^{*}\left(1-\left(\ln T^{*}\right) / \ln \left(R T^{*} / A^{*}\right)\right.} \leqslant\left(1+\frac{\ln T^{*}}{\alpha \ln T^{*}-s}\right) \sum_{\substack{e^{s-1}<A^{*} \leqslant e^{s} \\\left(A^{*}, T^{*}\right)=1}} \frac{1}{A^{*}} .
$$

Последнюю сумму мы оцениваем с помощью леммы 1, что дает неравенство

$$
\sum_{\substack{\left.s-1<A^{*} \leqslant e^{*} \\ e^{*}, T^{*}\right)=1}} \frac{1}{A^{*}\left(1-\left(\ln T^{*}\right) / \ln \left(R T^{*} / A^{*}\right)\right.} \leqslant\left(1+\frac{\ln T^{*}}{\alpha \ln T^{*}-s}\right) \frac{(1+o(1)) \varphi\left(T^{*}\right)}{T^{*}} .
$$

Вклад всех промежутков $\left(e^{s-1} ; e^{s}\right]$ в общую сумму по $A^{*}$ не превышает

$$
\begin{aligned}
\frac{\varphi\left(T^{*}\right)(1+o(1))}{T^{*}} \sum_{1 \leqslant s \leqslant\left\lceil\ln T^{*}\right\rceil}(1 & \left.+\frac{\ln T^{*}}{\alpha \ln T^{*}-s}\right) \\
\leqslant & \frac{\varphi\left(T^{*}\right)(1+o(1))}{T^{*}} \ln T^{*}\left(1+\sum_{1 \leqslant s \leqslant\left\lceil\ln T^{*}\right\rceil} \frac{1}{\alpha \ln T^{*}-s}\right) .
\end{aligned}
$$

Функщия $s \mapsto 1 /\left(\alpha \ln T^{*}-s\right)$ монотонно растет на промежутке [1; $\left.\left\lceil\ln T^{*}\right\rceil\right]$, а ее значения на этом промежутке есть $o(1)$, поэтому

$$
\begin{aligned}
\sum_{1 \leqslant s \leqslant\left\lceil\ln T^{*}\right\rceil} \frac{1}{\alpha \ln T^{*}-s} & \leqslant \int_{1}^{\left\lceil\ln T^{*}\right\rceil} \frac{d s}{\alpha \ln T^{*}-s}+o(1) \\
& =\left.\ln \left(\alpha \ln T^{*}-s\right)\right|_{\left[\ln T^{*}\right\rceil} ^{1}+o(1) \\
& =\ln \left(\alpha \ln T^{*}\right)-\ln \left((\alpha-1) \ln T^{*}\right)+o(1) \\
& =\ln \frac{\alpha}{\alpha-1}+o(1)
\end{aligned}
$$

Отсюда вытекает, что вклад всех промежутков $\left(e^{s-1} ; e^{s}\right]$ в общую сумму по $A^{*}$ не превышает

$$
\frac{\varphi\left(T^{*}\right)(1+o(1))}{T^{*}} \ln T^{*}\left(1+\ln \frac{\alpha}{\alpha-1}+o(1)\right)
$$

а соответствующий вклад в оценку числа $D_{0.2}$ из (40) не превышает

$$
\left(2+2 \ln \frac{\alpha}{\alpha-1}+o(1)\right) R n \ln T .
$$

Возьмем в качестве $\alpha$ корень уравнения

$$
2+2 \ln \frac{\alpha}{\alpha-1}=\alpha,
$$

то есть $\alpha=2,860356327 \ldots<2,860357$. Тогда получим, что число $D_{0.2}$ из (39) удовлетворяет оценке (37) и доказательство утверждения 2 леммы завершается.

Доказательство утверждения 3 легко вытекает из доказательства утверждения 2. Пусть сначала все $\xi_{i}$ равны 1 . Обозначим через $\Theta_{0.1}$ множество чисел вида $\theta_{0} \theta_{1}$, удовлетворяющих условиям леммы. Все отличие от предыдущего состоит в том, что $\Theta_{0}, \Theta_{1}$ и $D_{1}$ 
теперь исключаются из рассмотрения, а на $D_{0}$ налагается условие $0<D_{0} \in \operatorname{den} \Theta_{0,1}$. Заметим, что

$$
\begin{aligned}
\prod_{i \in\left\{i_{1} \ldots, i_{M}\right\}} \frac{\left(x_{i} ; T\right)_{a_{i}}^{A_{i}}}{\left(A_{i}-a_{i}\right) !} & =T^{\sum_{i \in\left\{i_{1} \ldots, i_{M}\right\}}\left(A_{i}-a_{i}\right)} \prod_{i \in\left\{i_{1} \ldots, i_{M}\right\}}\left(\begin{array}{c}
A_{i} \\
a_{i}
\end{array}\right) \\
& =T^{\sum_{j \in\left\{j_{1}, \ldots, j_{N}\right\}}\left(B_{j}-b_{j}\right)} \prod_{i \in\left\{i_{1}, \ldots, i_{M}\right\}}\left(\begin{array}{c}
A_{i} \\
a_{i}
\end{array}\right),
\end{aligned}
$$

поэтому $\theta_{0} \theta_{1}$ можно представить в виде

$$
\theta_{0} \theta_{1}=\prod_{i \in\left\{i_{1}, \ldots, i_{M}\right\}}\left(\begin{array}{c}
A_{i} \\
a_{i}
\end{array}\right) \prod_{j \in\left\{j_{1}, \ldots, j_{N}\right\}} \frac{T^{B_{j}-h_{j}}}{\pi_{j}} .
$$

Это показывает, что можно изменить определение числа $D_{0.0}$, взяв вместо (38)

$$
D_{0.0}=\prod_{q \mid T} q^{n\lfloor(\ln (R T)) / \ln q\rfloor} .
$$

Тем же рассуждением, что и выше, несложно показать, что $v_{q}\left(D_{0,0} \theta_{0} \theta_{1}\right) \geqslant 0$ для всех $\theta_{0} \theta_{1} \in \Theta_{0,1}$ и $q \mid T$, а оценку (35) можно заменить оценкой $\ln D_{0.0}=o(R n \ln T)$. Для числа $D_{0.2}$ теперь достаточно требовать выполнения не (37), а неравенства $\ln D_{0.2} \leqslant(\alpha+1+o(1)) R n \ln T$, где в качестве $\alpha$ нужно взять корень уравнения

$$
1+2 \ln \frac{\alpha}{\alpha-1}=\alpha,
$$

что дает $\alpha=2,2069956422 \ldots$ Значит, $\ln D \leqslant 3,207 R n \ln T$ при достаточно большом $T$, что и требовалось доказать.

Рассмотрим второй случай, когда $\left|\left\{j=1, \ldots, n: d \mid y_{j}\right\}\right| \leqslant n / d$ для всех $d \mid T$. Мы возвращаемся к ситуации утверждения 2 , но число $D_{0.0}$ из (38) оценим точнее. Пусть

$$
t_{q}(v)=\left|\left\{j=1, \ldots, n: v_{q}\left(y_{j}\right) \geqslant v\right\}\right| .
$$

Ясно, что $t_{q}(v)-t_{q}(v+1)$ есть число тех $j$, для которых $v_{q}\left(y_{j}\right)=v$, поэтому

$$
\begin{aligned}
\ln D_{0.0} \leqslant R \sum_{\substack{q \mid T \\
u=v_{q}(T)}} \ln q & \left(\sum_{1 \leqslant v<u} v\left(t_{q}(v)-t_{q}(v+1)\right)+\sum_{v \geqslant u} u\left(t_{q}(v)-t_{q}(v+1)\right)\right) \\
& +n \ln (R T) \sum_{q \mid T} 1=R \sum_{\substack{q \mid T \\
u=v_{q}(T)}} \ln q \sum_{1 \leqslant v \leqslant u} t_{q}(v)+O(n \ln R \ln T) .
\end{aligned}
$$

По условию $t_{q}(v) \leqslant n / q^{v}$, следовательно,

$$
\begin{aligned}
\ln D_{0.0} & \leqslant R n \sum_{\substack{q \mid T \\
u=v_{q}(T)}} \ln q \sum_{1 \leqslant v \leqslant u} \frac{1}{q^{v}}+o(R n \ln T) \leqslant R n \sum_{q \mid T} \frac{\ln q}{q-1}+o(R n \ln T) \\
& =O(R n \ln \ln T)+o(R n \ln T)=o(R n \ln T)
\end{aligned}
$$

(в предпоследнем переходе использована оценка (33)).

Это показывает, что число $\alpha$ можно выбрать в точности тем же, что и в предыдущем случае, чем и завершается доказательство леммы. 


\section{4. Доказательство теоремы}

Пусть $q$ - простое число. Ниже мы используем следующие стандартные обозначения: $\mathbf{Q}_{q}$ - замыкание $\mathbf{Q}$ по метрике $|x|_{q}=q^{-v_{q}(x)}$, где $v_{q}(x)-q$-показатель числа $x$ в $\mathbf{Q}$ (который продолжается до показателя в $\left.\mathbf{Q}_{q}\right) ; \mathbf{Z}_{q}$ - замыкание $\mathbf{Z}$ в $\mathbf{Q}_{q}$.

Наша ближайшая цель состоит в том, чтобы заменить число $\lambda$ из формулировки теоремы на

$$
\mu=\left(1-p^{E} / a\right)^{1 / T} \in \mathbf{Z}_{p} .
$$

Для этого достаточно показать, что $\lambda \equiv \mu(\bmod W)$ в $\mathbf{Z}_{p}$.

Разлагая оба числа $\lambda$ и $\mu$ в биномиальный ряд, видим, что достаточно доказать справедливость неравенства

$$
v_{p}\left(\left(\begin{array}{c}
1 / T \\
f
\end{array}\right)-\left(\begin{array}{c}
T^{\prime} \\
f
\end{array}\right)\right)+E f \geqslant v_{p}(W)
$$

при любом $f>0$. В силу тождества

$$
\left(\begin{array}{c}
X+Y \\
f
\end{array}\right)=\sum_{i, j \geqslant 0, i+j=f}\left(\begin{array}{l}
X \\
i
\end{array}\right)\left(\begin{array}{l}
Y \\
j
\end{array}\right)
$$

для биномиальных коэффициентов [4] это сводится к доказательству того, что

$$
v_{p}\left(\left(\begin{array}{c}
T^{\prime} \\
i
\end{array}\right)\right)+v_{p}\left(\left(\begin{array}{c}
\omega W \\
j
\end{array}\right)\right)+E(i+j) \geqslant v_{p}(W)
$$

при условии, что $i \geqslant 0, j \geqslant 1$ и $\omega \in \mathbf{Z}_{p}$. Но последнее верно, потому что

$$
\left(\begin{array}{c}
\omega W \\
j
\end{array}\right)=\frac{\omega W}{j}\left(\begin{array}{c}
\omega W-1 \\
j-1
\end{array}\right)
$$

при $j \geqslant 1$, и следовательно,

$$
v_{p}\left(\left(\begin{array}{c}
\omega W \\
j
\end{array}\right)\right) \geqslant v_{p}(W)-v_{p}(j) .
$$

Далее мы доказываем теорему в терминах числа $\mu$. Мы используем следующую хорошо известную конструкцию (см. [6]), носящую название приближений Эрмита-Паде для совокупности функций $(1-z)^{\omega_{j}}, j=1, \ldots, L$.

Пусть $\rho=\lceil F /(E L)\rceil$ - число из теоремы, $\delta_{i, j}$ - символ Кронекера, $\rho_{i, j}=\rho+\delta_{i, j}$. Рассмотрим комплексный интеграл

$$
J_{i}(z)=\frac{1}{2 \pi \sqrt{-1}} \int_{\mathscr{L}}(1-z)^{\zeta} \prod_{j=1}^{L} \prod_{s=0}^{\rho_{i, j}-1} \frac{d \zeta}{\zeta-\omega_{j}-s}
$$

где $\mathscr{L}$ - контур, охватывающий все полюсы подынтегральной функции. Вычисляя его как сумму вычетов в конечных точках, получим, что

$$
J_{i}(z)=\sum_{j=1}^{L}(1-z)^{\omega_{j}} P_{i, j}(z),
$$


где

$$
\begin{aligned}
P_{i, j}(z) & =\sum_{s=0}^{\rho_{i, j}-1}(1-z)^{s} p_{i, j, s}, \\
p_{i, j, s} & =\prod_{\substack{1 \leqslant J \leqslant L \\
0 \leqslant S \leqslant \rho_{i, j}-1 \\
(J, S) \neq(j, s)}} \frac{1}{\omega_{j}+s-\omega_{J}-S} .
\end{aligned}
$$

С другой стороны, вычет в $\infty$ есть

$$
\sum_{\substack{\mu_{0} \geqslant 0, \mu_{j, s} \geqslant 0, j=1, \ldots, L, s=0 \ldots, \rho_{i, j}-1 \\ \mu_{0}-\sum_{j, s} \mu_{j . s}=\sum_{j} \rho_{i, j}-1=\rho L}} \frac{(\ln (1-z))^{\mu_{0}}}{\nu_{0} !} \prod_{\substack{1 \leqslant j \leqslant L \\ 0 \leqslant s \leqslant \rho_{i, j}-1}}\left(\omega_{j}+s\right)^{\mu_{j . s}} .
$$

В последней сумме суммирование ведется по всем $\mu_{0} \geqslant 0$ и $\mu_{j, s} \geqslant 0$ для $j=1, \ldots, L$, $s=0, \ldots, \rho_{i, j}-1$, удовлетворяющим равенству $\mu_{0}-\sum_{j, s} \mu_{j, s}=\rho L$, при этом порядок нуля в точке $z=0$ слагаемого, соответствующего $\mu_{0}=\rho L, \mu_{j, s}=0$ (для любых $j, s$ ), строго меньше порядка нуля всех прочих слагаемых и равен $\rho L$. Значит, порядок нуля интеграла $J_{i}(z)$ равен $\rho L$ и $J_{i}(z) \not \equiv 0$ для $i=1, \ldots, L$.

Теперь возьмем $\omega_{j}=j / T, j=1, \ldots, L$ и оценим сверху максимум модуля получающихся чисел, если домножить все $p_{i, j, s}$ на их наименьший общий знаменатель. Предварительно выделим в $p_{i, j, s}$ два множителя

$$
\begin{aligned}
& p_{i, j . s}^{\prime}=\prod_{\substack{1 \leqslant J \leqslant L \\
J \neq j, 0 \leqslant S \leqslant \rho_{i, J}-1}} \frac{1}{\omega_{j}+s-\omega_{J}-S}, \\
& p_{i, j, s}^{\prime \prime}=\prod_{\substack{0 \leqslant \rho_{i, j}-1 \\
S \neq s}} \frac{1}{s-S},
\end{aligned}
$$

так что $p_{i, j, s}=p_{i, j, s}^{\prime} p_{i, j, s}^{\prime \prime}$.

Относительно $p_{i, j, s}^{\prime \prime}$ проблем нет: все $\rho ! p_{i, j, s}^{\prime \prime}$ - целые и ограничены по модулю величиной $2^{\rho}$. В случае с числами $p_{i, j . s}^{\prime}$ применим лемму 3. Чтобы проверить ее условия, рассмотрим совокупность

$$
\Theta^{\prime}=\left\{(\rho !)^{L-1}\left|p_{i, j, s}^{\prime}\right|: i=1, \ldots, L, j=1, \ldots, L, s=0,1, \ldots, \rho_{i, j}-1\right\} .
$$

Ясно, что $\Theta^{\prime}$ лежит в $\Theta$, если взять (в обозначениях леммы 3)

$$
\begin{aligned}
m & =L, & n & =2 L-2, \quad R=\rho+1, \quad T=T, \\
\xi_{i} & =1, & i & =1, \ldots, L, \\
\eta_{j} & =j / T, & j & =1, \ldots, L-1, \\
\eta_{j+L-1} & =1-j / T, & j & =1, \ldots, L-1
\end{aligned}
$$

(относительно последнего условия леммы заметим, что в нашем случае всегда

$$
\sum_{j=j_{1} \ldots . j_{N}}\left(B_{j}-b_{j}\right) \in[\rho(L-1) ; \rho(L-1)+1],
$$


этим оправдывается возможность введения поправочного множителя $(\rho !)^{L-1}$, то есть возможность считать, что $M=L-1$ или $M=L$ и для всех $i=i_{1}, \ldots, i_{M}$ за исключением, быть может, одного имеют место равенства $A_{i}=\rho, a_{i}=0$.)

Из первого утверждения леммы 3 следует, что

$$
\ln \max \Theta^{\prime}=n \ln T+o(R n \ln T) .
$$

Для оценки минимума $D^{\prime}$ абсолютных величин ненулевых элементов множества $\operatorname{den}\left(\Theta^{\prime}\right)$ мы воспользуемся доказательством второй части утверждения 3 леммы 3 . Если перейти к обозначениям этого доказательства, то для $D_{0,0}$ можно написать оценку

$$
\ln D_{0,0}=o(R n \ln T)
$$

(потому что выполнено предположение о числе элементов множества $\left\{j: d \mid y_{j}\right\}$ для $d \mid T)$, а число $D_{1}$ из (32) можно заменить на $T^{-\rho(L-1)}$, потому что все $\theta_{1} \in \Theta_{1}$ делятся на $T^{\rho(L-1)}$. Тем самым, мы получаем два неравенства

$$
\ln D_{0} \leqslant 3,206996 R n \ln T, \quad \ln D_{1}=-\rho(L-1) \ln T,
$$

откуда с учетом оценки $\ln D_{2}=O(R n)$ вытекает, что

$$
\begin{aligned}
\ln D^{\prime} & \leqslant 3,206996 R n \ln T-(R-1) \frac{n}{2} \ln T+O(R n) \\
& =2,706996 R n \ln T+O(R n+n \ln T) .
\end{aligned}
$$

В обозначениях теоремы

$$
\ln D^{\prime} \leqslant 5,413992 \rho(L-1) \ln T+O(\rho L+L \ln T) .
$$

Соединяя это с оценкой (42) для $\ln \max \Theta^{\prime}$, получим, что

$$
\ln \max D^{\prime} \Theta^{\prime} \leqslant 5,414 \rho(L-1) \ln T+O(L \ln T)
$$

при достаточно большом $T$.

Покажем, что если число $T$ достаточно велико, то в (43) можно убрать слагаемое $O(L \ln T)$. При $\rho>\ln T$ это ясно непосредственно, так как мы имеем право уменьшить константу 5,414 до 5,413992 , при этом величина $L \ln T$ будет асимптотически мала по сравнению с $\rho(L-1) \ln T$. Если же $\rho \leqslant \ln T$, то из неравенства (42) и доказательства утверждения 2 леммы 3 (оценка $D_{0}$ ) следует более сильная, чем (43), оценка

$$
\begin{aligned}
\ln \max D^{\prime} \Theta^{\prime} & \leqslant(1+o(1)) R n \ln T+n \ln T \\
& =(2+o(1)) \rho(L-1) \ln T+4(L-1) \ln T,
\end{aligned}
$$

так как при рассмотрении случая $\sigma=0$ мы можем ограничиться величиной $D_{0}$ из (34) с $\alpha=o(1)$. Но $(2+o(1)) \rho+4<5,414 \rho$ при всех $\rho \geqslant 2$ и достаточно большом $T$. Заметим, что мы можем изначально предполагать, что $\rho>L$ (следовательно, $\rho \geqslant 3$ ) ввиду замечания 2 , сделанного после формулировки теоремы (при $\rho \leqslant L$ утверждение теоремы теряет смысл). Итак,

$$
\ln \left|p_{i, j, s}^{*}\right| \leqslant 5,414 \rho(L-1) \ln T, \quad p_{i, j, s}^{*}=D^{\prime}(\rho !)^{L} p_{i, j, s},
$$

если $T$ достаточно велико. 
Теперь положим

$$
\begin{aligned}
P_{i, j}^{*}(z) & =\sum_{s=0}^{\rho_{i, j}-1}(1-z)^{s} p_{i, j . s}^{*}, \\
J_{i}^{*}(z) & =\sum_{j=1}^{L}(1-z)^{\omega_{j}} P_{i, j}^{*}(z) .
\end{aligned}
$$

По определению ряды $J_{i}^{*}(z)$ имеют целые коэффициенты в поле $\mathbf{Q}_{p}$ и первый ненулевой коэффициент у них стоит при степени $z^{\rho L}$. Далее, определитель

$$
\Delta^{*}(z)=\operatorname{det}\left(P_{i, j}^{*}(z)\right)
$$

матрицы $\left(P_{i, j}^{*}\right)$ размера $L \times L$ не равен 0 для $z \neq 0$, так как, с одной стороны, этот определитель есть многочлен от $z$ степени $\rho L$ (что легко установить, рассмотрев старшие члены многочленов $P_{i, j}^{*}(z)$ ), а с другой стороны, он делится на $z^{\rho L}$ (поскольку первый столбец определителя $\Delta^{*}(z)$ можно сложить с соответствующей линейной комбинацией остальных столбцов и получить на $i$-й позиции степенной ряд $(1-z)^{-1 / T} J_{i}^{*}(z)$, а все такие ряды делятся на $z^{\rho L}$ ).

Теперь мы вплотную приблизились к доказательству теоремы.

Предположим, что $\bar{h}=\left(h_{1}, \ldots, h_{L}\right)$ - ненулевой набор целых чисел, на котором достигается минимум $e_{L}(\lambda)$. Положим $h_{i, j}^{*}=a^{\rho} P_{i, j}^{*}\left(p^{E} / a\right)$ для всех $i, j$. Числа $h_{i, j}^{*}$ целые и при достаточно большом $T$ ограничены сверху величиной

$$
H^{*}=p^{\rho E} T^{5.414 \rho(L-1)},
$$

потому что $\ln L$ асимптотически мал по сравнению с $\rho(L-1) \ln T)$ и то же касается евклидовых длин векторов

$$
\bar{h}_{i}^{*}=\left(h_{i, 1}^{*}, \ldots, h_{i, L}^{*}\right)
$$

Линейные комбинации

$$
\begin{aligned}
\sum_{j=1}^{L} h_{i, j}^{*} \mu^{j} & =\sum_{j=1}^{L} a^{\rho} P_{i, j}^{*}\left(p^{E} / a\right)\left(1-p^{E} / a\right)^{j / T} \\
& =a^{\rho} J_{i}^{*}\left(p^{E} / a\right), \quad i=1, \ldots, L
\end{aligned}
$$

есть целые числа поля $\mathbf{Q}_{p}$, делящиеся на $W$ (потому что $J_{i}^{*}(z)$ делятся на $z^{\rho L}$, а $\rho E L \geqslant F=v_{p}(W)$ по определению числа $\left.\rho\right)$.

Определитель $\Delta^{*}$, строками которого являются векторы (46), не равен нулю. Заменим в $\Delta^{*}$ одну из строк строкой $\bar{h}$, так чтобы полученный определитель (обозначим его $\Delta_{1}^{*}$ ) также не был равен нулю. Из тех же соображений, что и выше (рассуждение о замене первого столбца определителя на его сумму с линейной комбинацией остальных) вытекает, что $\Delta_{1}^{*}$ - целое число, делящееся на $W$. Следовательно, $\left|\Delta_{1}^{*}\right| \geqslant p^{F}$. С другой стороны, из неравенства Адамара [4] вытекает верхняя оценка

$$
\left|\Delta_{1}^{*}\right| \leqslant e_{L}(\lambda) H^{* L-1} .
$$


Сравнивая эти оценки и учитывая (45), находим, что

$$
\begin{aligned}
e_{L}(\lambda) & \geqslant\left(p^{\rho E} T^{5,414 \rho(L-1)}\right)^{-(L-1)} p^{F} \\
& =\left(p T^{5,414(L-1) / E}\right)^{-(L-1) \rho E} p^{F} .
\end{aligned}
$$

Из определения числа $\rho$ вытекает оценка $E<F /((\rho-1) L)$, откуда,

$$
\rho E(L-1)<F \frac{\rho(L-1)}{(\rho-1) L}=F\left(1-\frac{1}{L}+\frac{L-1}{L(\rho-1)}\right) .
$$

Таким образом,

$$
\begin{aligned}
e_{L}(\lambda) & \geqslant p^{F / L-(L-1) F /(L(\rho-1))} T^{-5,414(L-1)(1-1 / L)(1+1 /(\rho-1)) F / E}=W^{1 / L-(1-1 / L) \delta} \\
\delta & =\frac{1}{\rho-1}+\frac{5,414(L-1)(1+1 /(\rho-1)) \ln T}{E \ln p} .
\end{aligned}
$$

В выражении для $\delta$ скобку $1+1 /(\rho-1)$ можно убрать, пользуясь теми же соображениями, что и выше: при $\rho \geqslant \ln T$ потому, что константу 5,414 можно слегка уменьшить, а при $\rho \leqslant \ln T$ потому, что, как мы видели выше (см. (44)), величину $5,414 \rho(L-1) \ln T$, участвующую в выражении для $\delta(47)$, можно заменить на $((2+o(1)) \rho+4)(L-1) \ln T$. Ho

$$
\frac{(2+o(1)) \rho+4}{\rho-1}<5,414
$$

при $\rho \geqslant 3$ и достаточно большом $T$. Следовательно, число $\delta$ будет иметь тот же вид, что и в теореме, что и требовалось доказать.

\section{5. Числовой пример}

По техническим причинам мы были вынуждены ограничиться случаем $W<2^{31}$, поэтому рассматриваемый ниже пример весьма далек от той ситуации, при которой оценка теоремы обретает смысл. Тем не менее он позволяет составить первое впечатление о качестве предлагаемых датчиков.

Напомним, что ставится задача проверки последовательности (3) на близость к случайной, причем под близостью к случайной последовательности в данном случае имеется в виду успешное прохождение спектрального теста в сильной форме. Для того чтобы датчик (1) периода максимальной длины при данном $L$ проходил спектральный тест в сильной форме, должно выполняться неравенство $\gamma_{L}(\lambda) \geqslant 0.1$, где $\gamma_{L}(\lambda)$ определено в (5). В приводимой ниже таблице в качестве $\lambda$ берутся числа теоремы 1 с параметрами $p=211, E=1, F=T=4$ и $a=-1, \ldots,-40$. Данный выбор параметров можно считать относительно произвольным, учитывая, что мы стремились, с одной стороны, удержать модуль $W=p^{F}$ в границе $W<2^{31}$, а с другой стороны, сделать его (равно как и число $p^{E}$ в соответствии с замечанием 2 после формулировки теоремы) достаточно большим. Еще раз подчеркнем, что ввиду малости выбранных нами параметров $F, T$, $\rho=\lceil F /(E L)\rceil$ и $p^{E}$ и несоблюдения в достаточной мере требований замечания 2 , данный пример не следует считать иллюстрацией к доказанной теореме. Тем не менее оказывается, что из 40 рассматриваемых датчиков лишь для одного величина $\min _{L=2,3,4} \gamma_{L}(\lambda)$ меньше 0.1 (при $a=-3$ ). Остальные 39 датчиков успешно проходят спектральный тест в сильной форме. 
Таблица 1. $p=211, E=1, F=T=4$

\begin{tabular}{|c|c|c|c|c|c|c|c|c|}
\hline$a$ & -1 & -2 & -3 & -4 & -5 & -6 & -7 & -8 \\
\hline$\gamma_{2}(\lambda)$ & 2,7202 & 2,4124 & 0,0889 & 2,1585 & 2,2232 & 2,4230 & 2,7634 & 0,1300 \\
\hline$\gamma_{3}(\lambda)$ & 0,2361 & 0,2509 & 0,2664 & 0,2824 & 0,2992 & 0,3166 & 0,3346 & 0,3534 \\
\hline$\gamma_{4}(\lambda)$ & 3,8501 & 3,1470 & 1,8073 & 0,7334 & 1,0222 & 2,9986 & 3,6051 & 2,5416 \\
\hline \hline$a$ & -9 & -10 & -11 & -12 & -13 & -14 & -15 & -16 \\
\hline$\gamma_{2}(\lambda)$ & 2,5382 & 2,8393 & 1,9248 & 2,8457 & 0,3232 & 1,1774 & 1,5310 & 0,7534 \\
\hline$\gamma_{3}(\lambda)$ & 0,3729 & 0,3931 & 0,4140 & 0,4357 & 0,4581 & 0,4813 & 0,5054 & 0,5302 \\
\hline$\gamma_{4}(\lambda)$ & 2,1747 & 5,7575 & 2,9443 & 1,5077 & 0,8752 & 0,7034 & 0,8789 & 0,5195 \\
\hline \hline$a$ & -17 & -18 & -19 & -20 & -21 & -22 & -23 & -24 \\
\hline$\gamma_{2}(\lambda)$ & 2,7873 & 0,6983 & 2,0789 & 0,6314 & 0,8201 & 2,1168 & 1,2871 & 2,8480 \\
\hline$\gamma_{3}(\lambda)$ & 0,5558 & 0,5822 & 0,6095 & 0,6377 & 0,6667 & 0,6966 & 0,7274 & 0,7591 \\
\hline$\gamma_{4}(\lambda)$ & 0,6587 & 0,5135 & 0,5639 & 0,8365 & 1,4583 & 1,7307 & 2,7877 & 4,8531 \\
\hline \hline$a$ & -25 & -26 & -27 & -28 & -29 & -30 & -31 & -32 \\
\hline$\gamma_{2}(\lambda)$ & 0,2500 & 2,3900 & 1,4969 & 2,1231 & 1,0073 & 0,5040 & 2,7350 & 0,9527 \\
\hline$\gamma_{3}(\lambda)$ & 0,7918 & 0,8253 & 0,8599 & 0,8953 & 0,9318 & 0,9692 & 1,0077 & 1,0472 \\
\hline$\gamma_{4}(\lambda)$ & 1,2001 & 3,1693 & 0,6815 & 0,4956 & 2,3755 & 3,8433 & 2,8082 & 2,1974 \\
\hline$a$ & -33 & -34 & -35 & -36 & -37 & -38 & -39 & -40 \\
\hline$\gamma_{2}(\lambda)$ & 3,2421 & 0,5183 & 1,5185 & 0,8822 & 0,5111 & 2,7612 & 1,6610 & 2,9962 \\
\hline$\gamma_{3}(\lambda)$ & 1,0877 & 1,1292 & 1,1718 & 1,2155 & 1,2603 & 1,3061 & 1,3531 & 1,4012 \\
\hline$\gamma_{4}(\lambda)$ & 3,3196 & 3,4023 & 2,9866 & 2,0221 & 0,5613 & 0,4693 & 0,3717 & 0,6776 \\
\hline
\end{tabular}

\section{Список литературы}

1. Виноградов И. М., Осиовы теории чисел. Наука, Москва, 1981.

2. Дэвенпорт Г., Мультипликативпая теория чисел. Наука, Москва, 1971.

3. Кнут Д., Искусство программировапия для ЭВМ. т. 2: Получислениые алгоритмы. Мир, Москва, 1977.

4. Корн Г., Корн Т., Справочиик по математике. Наука, Москва, 1973.

5. Прахар К., Распределепие простых чисел. Мир, Москва, 1967.

6. Chudnovsky G. V., On the method of Tue-Siegel. Ann. Math. (1983) 117, 325-382.

7. Hata M., Legendre type polynomials and irrationality measures. J. Reine und Angewandte Math. (1990) 407, 99-125.

8. Hata M., A lower bound for rational approximations to $\pi$. J. Number Theory (1993) 43, 51-67.

9. Hata M., Rational approximations to $\pi$ and some other numbers. Acta Arithmetica (1993) 153, 335-349.

10. Heimonen A., On effective irrationality measures for some values of certain hypergeometric functions. Diss. Oulu Univ., Oulu, 1997.

Статья поступила 26.02.2003. 